\title{
A mathematical model for heating and evaporation of a multi-component liquid film
}

\author{
S.S. Sazhin*, O. Rybdylova, C. Crua \\ Advanced Engineering Centre, \\ School of Computing, Engineering and Mathematics, \\ University of Brighton, Brighton, BN2 4GJ, UK
}

\begin{abstract}
A new model for heating and evaporation of a multi-component liquid film, based on the analytical solutions to the heat transfer and species diffusion equations inside the film, is suggested. The Dirichlet boundary condition is used at the wall and the Robin boundary condition is used at the film surface for the heat transfer equation. For the species diffusion equations, the Neumann boundary conditions are used at the wall, and Robin boundary conditions are used at the film surface. The convective heat transfer coefficient is assumed to be constant and the convective mass transfer coefficient is inferred from the ChiltonColburn analogy. The model is validated using the previously published experimental data for heating and evaporation of a film composed of mixtures of isooctane/3-methylpentane (3MP). Also, it is applied to the analysis of heating and evaporation of a film composed of a 50\%/50\% mixture of heptane and hexadecane in Diesel engine-like conditions.
\end{abstract}

Keywords: Liquid film, heating, evaporation, Diesel engine, multi-component, analytical solutions

\section{Nomenclature}

c specific heat capacity

$c_{\mu} \quad$ constant equal to 0.09

D diffusion coefficient

$f_{n} \quad$ parameter introduced in (4)

$h \quad$ convection heat transfer coefficient

$h_{0} \quad h \delta_{0} / k_{l}$

$k \quad$ thermal conductivity

$L \quad$ specific heat of evaporation

Le Lewes number

\footnotetext{
${ }^{*}$ Corresponding author: e-mail S.Sazhin@brighton.ac.uk.
} 


\begin{tabular}{|c|c|}
\hline$M$ & molar mass \\
\hline$\dot{m}_{f}$ & evaporation mass flux \\
\hline$N$ & total number of evaporating species \\
\hline $\mathrm{Nu}$ & Nusselt number \\
\hline$p$ & pressure or parameter introduced in Equation (20) \\
\hline $\operatorname{Pr}$ & Prandtl number \\
\hline$q$ & heat flux \\
\hline$q_{n}$ & parameter introduced in Equation (4) \\
\hline$q_{Y n}$ & parameter introduced in Equation (27) \\
\hline$R_{\mathrm{u}}$ & universal gas constant \\
\hline $\operatorname{Re}$ & Reynolds number \\
\hline $\mathrm{Sc}$ & Schmidt number \\
\hline$t$ & time \\
\hline$T$ & temperature \\
\hline$u$ & parameter defined by Equation (14) \\
\hline$u^{*}$ & friction velocity \\
\hline$v_{n}$ & eigenfunction defined by Equation (23) \\
\hline$x$ & distance from the wall \\
\hline$X$ & $x / \delta_{0}$ or molar fraction \\
\hline$y$ & distance from the surface of the film \\
\hline$Y$ & mass fraction \\
\hline \multicolumn{2}{|c|}{ Greek symbols } \\
\hline$\delta$ & film thickness \\
\hline$\epsilon_{i}$ & parameter defined by (13) \\
\hline$\Theta$ & function introduced in (19) \\
\hline$\kappa$ & thermal diffusivity \\
\hline$\kappa_{\delta 0}$ & $k_{l} /\left(c_{l} \rho_{l} \delta_{0}^{2}\right)$ \\
\hline$\lambda_{n}$ & eigenvalues \\
\hline$\mu$ & dynamic viscosity \\
\hline$\nu$ & kinematic viscosity \\
\hline$\rho$ & density \\
\hline
\end{tabular}




$\begin{array}{ll}\text { Subscripts } & \\ \text { a } & \text { ambient } \\ \text { eff } & \text { effective } \\ \text { e } & \text { evaporation } \\ \text { f } & \text { film } \\ \mathrm{g} & \text { gas } \\ i & \text { species } \\ \text { in } & \text { inner } \\ \text { l } & \text { liquid phase } \\ \mathrm{m} & \text { mass transfer } \\ \text { out } & \text { outer } \\ \mathrm{p} & \text { constant pressure } \\ \mathrm{s} & \text { surface of the film } \\ \mathrm{T} & \text { turbulent } \\ \mathrm{v} & \text { vapour phase } \\ \mathrm{w} & \text { wall } \\ 0 & \text { value at the beginning of a time step or initial value }\end{array}$

\section{Introduction}

The importance of modelling liquid film heating and evaporation in various engineering applications is well known [1]. Recently, most of the attention has been focused on the application of these models to the analysis of the processes in internal combustion engines (see [2] and the references therein).

The formation of liquid films in engine combustion systems is known to lead to detrimental processes, including the formation of smoke-inducing piston fuel films in spark-ignited engines [3], and the initiation of fuel films on the surface of Diesel injectors [4]. The accumulation of deposits inside and on the surface of fuel injector nozzles is associated with reduced engine performance and lifetime. This reduction in performance is known to manifest in a variety of ways including increased acoustic and pollutant emissions [5, 6, 7]. The deposits can also reduce the hydraulic diameter of the nozzle orifices, resulting in a reduction in the quantity of injected fuel and reduced repeatability of injection $[7,8,9]$, all of which cause a reduction in engine power [10]. The deposits can also increase cavitation, which can then lead to further coking of the nozzle [11]. Injector needle sticking can occur and eventually lead to injector failure [12, 13]. Accurate mathematical modelling of the heating and evaporation of multi-component liquid films can help us to 
improve our understanding of the formation and decomposition processes of fuel films, and lead to the development of more efficient prevention and control strategies.

The modelling of heating and evaporation of liquid films has been considered in a number of papers some of which are reviewed in [2]. The simplest model of these processes is based on the assumption that the liquid is well mixed and the thermal diffusion inside it can be considered infinitely fast (zero-dimensional model). In [14] the temperature gradients inside the liquid film were taken into account, but based on a rather simplistic assumption that the temperature distribution can be approximated by the piecewise linear function (linear temperature model). The model based on the assumption of a more complex polynomial distribution of temperature inside the liquid film is known as the quasi-dimensional model [2]. Finally the model based on the rigorous solution to the heat conduction equation inside the liquid film and the assumption that temperature gradients in the direction perpendicular to the wall are much larger than those along the wall is known as the one-dimensional model [2]. Our analysis is focused on the latter model, as the most general one.

In contrast to most previously suggested models of the phenomenon, we will take into account the presence of multiple components in the liquid film, which is typical for automotive fuels. Both thermal and species diffusion inside the liquid film will be taken into account. As in the case of the analysis of multi-component droplet heating and evaporation (see [15]), the model is based on the analytical solutions to the heat transfer and species diffusion equations. The film will be assumed to be thin which will allow us to use the one-dimensional model in which both temperature and liquid species mass fractions depend only on the distance from the wall.

We start our analysis with the case of mono-component liquid films (Section 2). In Section 3 this analysis is generalised to the case of multi-component liquid films. The solution algorithm is described in Section 4. The validation of the model against published experimental data and its application to the analysis of specific cases of bi-component fuel film heating and evaporation in engine-like conditions is described in Section 5. The main results of the paper are summarised in Section 6.

\section{Heating and evaporation of a liquid film (mono-component liquid)}

The analyses of the processes in liquid and gas phases, described within this model, are presented in the following subsections.

\subsection{Liquid phase}

Assuming that the gradients of temperature in the film in the direction perpendicular to the wall are much greater than those in the direction parallel to the wall, the heat conduction equation inside the film can be simplified to:

$$
\frac{\partial T}{\partial t}=\kappa_{1} \frac{\partial^{2} T}{\partial x^{2}},
$$


where $\kappa_{1}=k_{1} /\left(c_{1} \rho_{1}\right)$ is the liquid thermal diffusivity, $k_{1}, c_{1}$, and $\rho_{1}$ are the liquid thermal conductivity, specific heat capacity, and density, respectively, $x$ is the distance from the wall.

Equation (1) is expected to describe the heat conduction process in this film except in the vicinity of its edges. Also, in the case of thin films, the contribution of the convective term, ignored in Equation (1), is expected to be very small.

Following [16], we assume that the liquid temperature at the wall is equal to the constant wall temperature: $T(x=0, t)=T_{\mathrm{w}}$ (Dirichlet boundary condition).

Yan et al. [17] specified heat flux rather than temperature at the wall (Neumann boundary condition). This heat flux was estimated as $q_{\mathrm{w}}=k_{\mathrm{w}}\left(T_{\mathrm{out}}-T_{\mathrm{in}}\right) / \delta_{\mathrm{w}}$, where $k_{\mathrm{w}}$ is the thermal conductivity of the wall, $\delta_{\mathrm{w}}$ is the wall thickness, $T_{\text {out }}$ and $T_{\text {in }}$ are wall temperatures at the outer and inner boundaries. This approach to the estimation of the heat flux is applicable only in the case of steady state problems, which is not compatible with the modelling of the transient process in the liquid film. The rigorous approach to this problem would require a coupled solution for the liquid film and the wall similar to the one considered in [15] for spherical layers. To the best of our knowledge, this approach to the problem of liquid film heating and evaporation has not been investigated.

Following [16], the boundary condition at the surface of the liquid film $\left(x=\delta_{0}\right)$ is presented as:

$$
h\left(T_{\text {eff }}-T_{\mathrm{s}}\right)=\left.k_{1} \frac{\partial T}{\partial x}\right|_{x=\delta_{0}-0},
$$

where

$$
T_{\text {eff }}=T_{\mathrm{g}}+\frac{\rho_{\mathrm{l}} L \dot{\delta}_{0 e}}{h}
$$

the value of $\dot{\delta}_{0 e}$ (the derivative of the film thickness with respect to time), controlled by film evaporation (indicated by the additional subscript ${ }_{e}$ ), is taken from the previous time step, $L$ is the specific heat of evaporation, $T_{\mathrm{g}}$ and $T_{\mathrm{s}}$ are ambient gas and film surface temperatures, respectively. The value of $\dot{\delta}_{0 e}$ is estimated later (see Expression (9)).

Equation (2) is the energy balance equation at the surface of the film: heat transferred by convection from ambient gas to the surface is spent on the evaporation of the film and its heating. This equation describes the Robin boundary condition for Equation (1) at the surface of the film.

The above-mentioned boundary conditions are supplemented by the initial condition $T(t=0)=T_{0}(x)$.

Traditionally, the heat transfer equation inside the film, along with related equations, in most cases has been solved numerically (e.g. [18]). The authors of [16] derived an analytical solution to Equation (1) subject to the above-mentioned boundary and initial conditions. A simplified and slightly corrected version of this solution can be presented as follows, using notations different from those used in [16]:

$$
T(X, t)=T_{\mathrm{w}}+\frac{X h_{0}}{1+h_{0}}\left(T_{\mathrm{eff}}-T_{\mathrm{w}}\right)+\sum_{n=1}^{\infty} \exp \left[-\kappa_{\delta 0} \lambda_{n}^{2} t\right]\left[q_{n}+f_{n} h_{0}\left(T_{\mathrm{eff}}-T_{\mathrm{w}}\right)\right] \sin \left(\lambda_{n} X\right),
$$


where $X=x / \delta_{0}, h_{0}=h \delta_{0} / k_{1}, \kappa_{\delta 0}=k_{1} /\left(c_{1} \rho_{1} \delta_{0}^{2}\right)$,

$$
\begin{gathered}
q_{n}=\frac{1}{\left\|v_{n}\right\|^{2}} \int_{0}^{1}\left(T_{0}(X)-T_{\mathrm{w}}\right) \sin \left(\lambda_{n} X\right) \mathrm{d} X, \quad f_{n}=\frac{1}{\left\|v_{n}\right\|^{2}} \int_{0}^{1} f(X) \sin \left(\lambda_{n} X\right) \mathrm{d} X=-\frac{\sin \lambda_{n}}{\left\|v_{n}\right\|^{2} \lambda_{n}^{2}}, \\
f(X)=-X /\left(1+h_{0}\right),\left\|v_{n}\right\|^{2}=\frac{1}{2}\left(1-\frac{\sin 2 \lambda_{n}}{2 \lambda_{n}}\right)=\frac{1}{2}\left(1+\frac{h_{0}}{h_{0}^{2}+\lambda_{n}^{2}}\right), \lambda_{n} \text { are non-trivial solutions to the equation } \\
\lambda \cos \lambda+h_{0} \sin \lambda=0 .
\end{gathered}
$$

Note that there is a typo in Expression (7) of [16]: the sign before $f_{n}$ in their formula should be a minus. When deriving Equation (4) we considered that both $h_{0}$ and $T_{\text {eff }}$ remain constant during the time step. With these assumptions, the last term in Expression (7) of [16] should be zero.

Solution (4) is expected to be used at each time step in the numerical code. The values of temperature predicted by this equation at the end of the previous time step are used as the initial values for the following time step, with updated values of input parameters (e.g. thickness of the film and gas temperature).

Note that Solution (4) could be easily obtained from the corresponding solution for droplet heating and evaporation presented back in 2004 in [19] (see Appendix A of [15]).

\subsection{Gas phase}

To complete the analysis of the previous section, two parameters, $h$ and $\dot{\delta}_{0 e}$, need to be estimated. These parameters could be inferred from the gas phase model or from experimental data.

Following [16] we assume that the fuel vapour at the film surface is always saturated and the analysis of the evaporation process reduces to the analysis of vapour diffusion from the film surface to the ambient gas. In the case of evaporating droplets it is typically assumed that vapour mass fraction at an infinitely large distance from the droplet surface is zero [15]. In the case of an evaporating liquid film, however, this assumption would lead to unphysical infinitely large evaporation rates and cannot be used in our model. ${ }^{1}$

Following $[20,2]$, we start the analysis of the evaporation process with an estimation of the convection heat transfer coefficient $h$, taking into account the effects of turbulence. The latter could be determined based on the wall function for turbulent gas (the $k-\epsilon$ model was used for turbulence modelling) using the following formula $[14,20,2]$ :

$$
h= \begin{cases}\frac{\rho_{\mathrm{g}} c_{p g} u^{*} T \ln \left(T / T_{\mathrm{s}}\right)}{\left(2.1 \ln y^{+}+2.5\right)\left(T-T_{\mathrm{s}}\right)} & y^{+}>y_{c}^{+} \\ \frac{\rho_{\mathrm{g}} c_{p g} u^{*}}{\operatorname{Prg}_{\mathrm{g}} y^{+}} & y^{+} \leq y_{c}^{+},\end{cases}
$$

where $\rho_{\mathrm{g}}$ and $c_{p \mathrm{~g}}$ are density and specific heat capacity of the ambient gas taken at the reference temperature (the contribution of fuel vapour to these parameters is usually ignored), $u^{*}$ is the friction velocity defined as $u^{*}=c_{\mu}^{0.25} \sqrt{k_{\mathrm{T}}}, c_{\mu}=0.09, k_{\mathrm{T}}$ is the turbulent kinetic energy, $y_{\mathrm{c}}^{+}=11.05$ is the criterion of the laminar

\footnotetext{
${ }^{1}$ This has been overlooked in the model described in [2] (see their Section 2.2).
} 
turbulent transition, $\operatorname{Pr}_{\mathrm{g}}=\mu_{\mathrm{g}} c_{p \mathrm{~g}} / k_{\mathrm{g}}$ is the laminar Prandtl number, $\mu_{\mathrm{g}}$ and $k_{\mathrm{g}}$ are gas dynamic viscosity and thermal conductivity, respectively (as in the case of density and specific heat capacity, the effects of vapour on these parameters are usually ignored), $y^{+}=y c_{\mu}^{0.25} k_{\mathrm{T}}^{0.5} / \nu_{\mathrm{g}}, \nu_{\mathrm{g}}=\mu_{\mathrm{g}} / \rho_{\mathrm{g}}$ is the gas kinematic viscosity, $y$ is the distance from the surface of the film to the first mesh node near this surface, $T_{\mathrm{s}}$ is the temperature at the surface of the film, $T$ is the temperature at this mesh node (to be calculated using any conventional Computational Fluid Dynamics (CFD) code).

This approach to the estimation of $h$, however, seems rather awkward for practical applications. Alternatively, one might use one of the correlations for the gas phase Nusselt number $(\mathrm{Nu})$. These correlations predict that $\mathrm{Nu}$ is proportional to $\operatorname{Re}^{4 / 5}[21,17,20,2]$ and are not expected to be valid at small $\mathrm{Re}$, for which they predict that $h=0$. These approaches will not be used in our analysis and we will use the experimentally observed values of $h$, including those observed in Diesel engine-like conditions.

The results of experimental investigations of $h$ in Diesel engine-like conditions showed that this coefficient can vary from about $500 \mathrm{~W} /\left(\mathrm{m}^{2} \mathrm{~K}\right)$ to $5500 \mathrm{~W} /\left(\mathrm{m}^{2} \mathrm{~K}\right)[22]$. When testing our model in Diesel engine-like conditions we assume, following [17], that $h=2000 \mathrm{~W} /\left(\mathrm{m}^{2} \mathrm{~K}\right)$.

Once the value of $h$ has been estimated, the value of the mass transfer coefficient is estimated using the Chilton-Colburn analogy as [23]:

$$
h_{\mathrm{m}}=\frac{h}{\rho_{\mathrm{g}} c_{p \mathrm{~g}}} \mathrm{Le}^{-2 / 3},
$$

where $\mathrm{Le}=\mathrm{Sc} / \mathrm{Pr}_{\mathrm{g}}$ is the gas Lewis number, $\mathrm{Sc}=\mu_{\mathrm{g}} / \rho_{\mathrm{g}} D_{\mathrm{g}}$ is the Schmidt number, $D_{\mathrm{g}}$ is gas diffusivity. In the case of multi-component vapour, the diffusivities of all components are assumed to be the same, leading to a common Sc.

The convection mass transfer coefficient inferred from Equation (7) allows us to estimate the evaporation mass flux from the film surface as [21]:

$$
\dot{m}_{\mathrm{f}}=h_{\mathrm{m}}\left(\rho_{\mathrm{vs}}-\rho_{\mathrm{va}}\right) \text {, }
$$

where $\rho_{\mathrm{vs}}$ and $\rho_{\mathrm{va}}$ are the vapour density at the surface of the film and in ambient gas, respectively. In our analysis we assume that $\rho_{\mathrm{va}}=0$. The generalisation of the analysis to the case of non-zero $\rho_{\mathrm{va}}$ would have been straightforward provided that the value of this parameter had been specified (e.g. from a coupled CFD solution). This generalisation is beyond the scope of the current paper.

The value of $\dot{\delta}_{0 e}$ is estimated as:

$$
\dot{\delta}_{0 e}=-\left|\frac{\dot{m}_{\mathrm{f}}}{\rho\left(\bar{T}_{0}\right)}\right|,
$$

where $\bar{T}_{0}$ is the average temperature in the film. This formula is used in Expression (3) for $T_{\text {eff }}$.

Note that there is a typo in Equation (22) of [20] (the power of Le has the wrong sign; the corresponding Equation (21) of [2] is correct). The mass transfer coefficient used in [20,2] is normalised by gas density. 


\section{Heating and evaporation of a liquid film (multi-component liquid)}

All equations for heating mono-component films, derived in the previous section, remain valid for multicomponent films. In contrast to mono-component films, however, we need to take into account the effect of mutual diffusion of species as in the case of heating and evaporation of multi-component droplets [15]. The modelling of the diffusion of species in the liquid phase is discussed in Section 3.1. The implications of this diffusion for the processes in the gas phase are discussed in Section 3.2.

\subsection{Liquid phase}

Assuming that the gradients of species mass fractions in the film in the direction perpendicular to the wall are much greater than those in the direction parallel to the wall (cf. similar assumption about temperature gradients in Section 2.1), the species diffusion equation inside the film can be simplified to:

$$
\frac{\partial Y_{1, i}}{\partial t}=D_{1} \frac{\partial^{2} Y_{1, i}}{\partial x^{2}}
$$

where $D_{1}$ is the liquid diffusion coefficient (assumed to be the same for all species (cf. similar assumption made for the analysis of multi-component droplet heating and evaporation [15])), $x$ is the distance from the wall.

Equation (10) will be solved subject to the following boundary conditions at the outer surface of the film and at the wall:

$$
\begin{gathered}
\left.D_{1} \frac{\partial Y_{1, i}}{\partial x}\right|_{x=\delta_{0}-0}=\left|\dot{\delta}_{0 e}\right|\left(\left.Y_{1, i}\right|_{x=\delta_{0}}-\epsilon_{i}\right), \\
\left.\frac{\partial Y_{1, i}}{\partial x}\right|_{x=0}=0
\end{gathered}
$$

where $\left|\dot{\delta}_{0 e}\right|=\left|\dot{m}_{\mathrm{f}} / \rho_{\mathrm{l}}\right|=h_{m} \sum_{i=1}^{i=N} \rho_{\mathrm{vs} i} / \rho_{\mathrm{l}}$ is the evaporation flux from the surface of the film, $\rho_{\mathrm{vs} i}$ are densities of the $i$ th vapour species at the outer surface of the film, $\rho_{1}$ is the density of the mixture of liquid species, $N$ is the total number of evaporating species,

$$
\epsilon_{i}=\frac{Y_{\mathrm{vs}, i}}{\sum_{i=1}^{i=N} Y_{\mathrm{vs}, i}}=\frac{\rho_{\mathrm{vs}, i}}{\sum_{i=1}^{i=N} \rho_{\mathrm{vs}, i}},
$$

$Y_{\mathrm{vs}, i}$ and $\rho_{\mathrm{vs}, i}$ are the mass fraction and vapour density of the $i$ th vapour species at the outer surface of the film, respectively. The initial condition for Equation (10) is $Y_{1, i}(t=0)=Y_{10, i}$. Note that in our sign convention $\dot{m}_{\mathrm{f}}$ is positive, while $\dot{\delta}_{0 e}$ is negative.

The physical meaning of Equation (11) is the same as that used for the analysis of heating and evaporation of multi-component droplets (cf. Equation (5.9) in [15]). Equation (12) is the mathematical expression of the fact that species cannot diffuse through the wall.

To simplify our analysis further, we assume that $\epsilon_{i}=$ const. This assumption can be supported by the fact that Equation (10) will be solved over a relatively short time step. The main parameter which 
could influence the values of $\epsilon_{i}$ is the rate of change of temperature during the time step. This change in temperature is expected to be greatest during the heating up period of the film when its evaporation is expected to be at the weakest. During the strongest evaporation at high temperatures, this change in temperature is expected to be relatively small.

Introducing a new variable:

$$
u=Y_{1, i}-\epsilon_{i},
$$

Equation (10) and boundary conditions (11) and (12) can be rewritten as:

$$
\begin{gathered}
\frac{\partial u}{\partial t}=D_{1} \frac{\partial^{2} u}{\partial x^{2}}, \\
\frac{\partial u}{\partial x}-\left.\frac{\left|\dot{\delta}_{0 e}\right|}{D_{1}} u\right|_{x=\delta_{0}-0}=0, \\
\left.\frac{\partial u}{\partial x}\right|_{x=0}=0 .
\end{gathered}
$$

The initial condition for Equation (15) is presented as:

$$
u(t=0)=Y_{10, i}(x)-\epsilon_{i}=u_{0}(x),
$$

where the additional subscript ${ }_{0}$ indicates initial values.

One can see a similarity between Equations (15)-(18) and Equations (H.7)-(H.9) in [15] derived for the analysis of species diffusion in multi-component droplets, except that for the droplets it was assumed that $u$ at the centre of the droplet is zero (Dirichlet boundary condition) which is different from the corresponding boundary condition for the film (Equation (17), Neumann boundary condition). Hence, we need to find a new solution to Equation (15) rather than to adapt the one described in Appendix $\mathrm{H}$ of [15] to our problem.

As in Appendix H of [15] we look for the solution to (15) in the form:

$$
u \equiv u(t, x)=\sum_{n=0}^{\infty} \Theta_{n}(t) v_{n}(x),
$$

where $v_{n}(x)$ is the full set of non-trivial solutions to the equation:

$$
\frac{\partial^{2} v}{\partial x^{2}}+p v=0
$$

subject to the boundary conditions:

$$
\begin{gathered}
\left.\frac{\partial v}{\partial x}\right|_{x=0}=0 \\
\left.\left(\frac{\partial v}{\partial x}-\frac{\left|\dot{\delta}_{0 e}\right|}{D_{\mathrm{l}}} v\right)\right|_{x=\delta_{0}}=0
\end{gathered}
$$

Equation (20) with boundary conditions (21) and (22) is the well known Sturm-Liouville problem. Our first task is to find eigenvalues $p$ for this problem. The case $p=0$ leads to the trivial solution $v=0$ (cf. Appendix 
$\mathrm{H}$ of [15]). The cases $p<0$ and $p>0$ are considered in Appendix 1. The following set of eigenfunctions was obtained (see Equations (43) and (48)):

$$
v_{n}(x)= \begin{cases}\cosh \left(\lambda_{0} \frac{x}{\delta_{0}}\right) & n=0 \\ \cos \left(\lambda_{n} \frac{x}{\delta_{0}}\right) & n \geq 1,\end{cases}
$$

where $\lambda_{n}(n \geq 0)$ are the solutions to the following equations (see (42) and (47)):

$$
\operatorname{coth} \lambda_{0}=\frac{\lambda_{0} D_{1}}{\left|\dot{\delta}_{0 e}\right| \delta_{0}}, \quad \cot \lambda_{n}=-\frac{\lambda_{n} D_{1}}{\left|\dot{\delta}_{0 e}\right| \delta_{0}} \quad(n \geq 1) .
$$

Having substituted (19) into (15) we obtain:

$$
\sum_{n=0}^{\infty} \Theta_{n}^{\prime}(t) v_{n}(x)=D_{1} \sum_{n=0}^{\infty} \Theta_{n}(t) v_{n}^{\prime \prime}(x)
$$

where

$$
\Theta_{n}^{\prime}(t)=\frac{\mathrm{d} \Theta_{n}(t)}{\mathrm{d} t}, \quad v_{n}^{\prime \prime}(x)=\frac{\mathrm{d}^{2} v_{n}(x)}{\mathrm{d} x^{2}} .
$$

Since the expansion in a series with respect to $v_{n}$ (Fourier series) is unique, Equation (24) is satisfied only when it is satisfied for each term in this expansion. Remembering that

$$
v_{0}^{\prime \prime}=\left(\frac{\lambda_{0}}{\delta_{0}}\right)^{2} v_{0} \quad \text { and } \quad v_{n}^{\prime \prime}=-\left(\frac{\lambda_{n}}{\delta_{0}}\right)^{2} v_{n} \quad(n \geq 1)
$$

this implies that:

$$
\begin{gathered}
\Theta_{0}^{\prime}(t)=D_{1}\left(\frac{\lambda_{0}}{\delta_{0}}\right)^{2} \Theta_{0}(t), \\
\Theta_{n}^{\prime}(t)=-D_{1}\left(\frac{\lambda_{n}}{\delta_{0}}\right)^{2} \Theta_{n}(t), \quad n \geq 1 .
\end{gathered}
$$

To find initial conditions for (25) and (26) we recall that $u(t=0)=u_{0}(x)$ (see (18)). Expansion of $u_{0}(x)$ in the Fourier series with respect to $v_{n}$ gives:

$$
u_{0}(x)=\sum_{n=0}^{\infty} q_{Y n} v_{n}(x)
$$

where

$$
q_{Y n}=\frac{1}{\left\|v_{n}\right\|^{2}} \int_{0}^{\delta_{0}} u_{0}(x) v_{n}(x) \mathrm{d} x .
$$

Comparing (24) and (27) one can see that the initial conditions for (25) and (26) can be presented as:

$$
\Theta_{n}(t=0)=q_{n}, \quad n \geq 0 .
$$

This leads us to the following solutions to Equations (25) and (26):

$$
\Theta_{0}(t)=q_{Y 0} \exp \left[D_{1}\left(\frac{\lambda_{0}}{\delta_{0}}\right)^{2} t\right],
$$




$$
\Theta_{n}(t)=q_{Y n} \exp \left[-D_{1}\left(\frac{\lambda_{n}}{\delta_{0}}\right)^{2} t\right]
$$

where $n \geq 1$.

Having substituted (29) and (30) into (19), and remembering (23), we obtain:

$$
u=q_{Y 0} \exp \left[D_{1}\left(\frac{\lambda_{0}}{\delta_{0}}\right)^{2} t\right] \cosh \left(\lambda_{0} \frac{x}{\delta_{0}}\right)+\sum_{n=1}^{\infty} q_{Y n} \exp \left[-D_{1}\left(\frac{\lambda_{n}}{\delta_{0}}\right)^{2} t\right] \cos \left(\lambda_{n} \frac{x}{\delta_{0}}\right) .
$$

Remembering the definition of $u$ (see (14)), the final expression for $Y_{1, i}(t, x)$, satisfying boundary conditions (11), (12) and the corresponding initial condition, can be presented as:

$$
Y_{1, i}(t, x)=q_{Y 0} \exp \left[D_{1}\left(\frac{\lambda_{0}}{\delta_{0}}\right)^{2} t\right] \cosh \left(\lambda_{0} \frac{x}{\delta_{0}}\right)+\sum_{n=1}^{\infty} q_{Y n} \exp \left[-D_{1}\left(\frac{\lambda_{n}}{\delta_{0}}\right)^{2} t\right] \cos \left(\lambda_{n} \frac{x}{\delta_{0}}\right)+\epsilon_{i} .
$$

Growth of $Y_{1, i}(t, x)$ with time is always restricted by the physical condition $0 \leq Y_{1, i}(t, x) \leq 1$ (cf. Equation (5.18) of [15], describing species diffusion in multi-component droplets; the latter equation was verified against results based on the rigorous numerical solution of the species diffusion equation [24]). Equation (32) is valid only for short time steps when $\dot{\delta}_{0 e}$ and all other input parameters are assumed constant.

\subsection{Gas phase}

Once the mass fractions of the liquid components at the surface of the film have been found, the partial pressures of vapour components at the film surface can be inferred from Raoult's law (the activity coefficient is assumed equal to 1 ):

$$
p_{\mathrm{vs}, i}=X_{\mathrm{ls}, i} p_{\mathrm{v}, i}^{*},
$$

where $X_{\mathrm{ls}, i}$ is the molar fraction of the $i$ th species in the liquid near the film surface, $p_{\mathrm{v}, i}^{*}$ is the partial vapour pressure of the $i$ th species in the case when $X_{1, i}=1$.

The values of $X_{\mathrm{ls}, i}$ and $Y_{\mathrm{ls}, i}$ are linked by the following equation:

$$
X_{\mathrm{ls}, i}=\frac{\frac{Y_{\mathrm{ls}, i}}{M_{i}}}{\sum_{i}\left(\frac{Y_{\mathrm{ls}, i}}{M_{i}}\right)}
$$

where $M_{i}$ is the molar mass of species $i$. Equation (34) follows from the definition of the mass fraction:

$$
Y_{\mathrm{ls}, i}=\frac{X_{\mathrm{ls}, i} M_{i}}{\sum_{i} X_{\mathrm{ls}, i} M_{i}} .
$$

The values of $p_{\mathrm{v}, i}^{*}$ depend on gas temperatures in the immediate vicinity of the film surface and can be obtained using the Antoine equation. The relations relevant to Diesel fuel components are summarised in [25].

Once the values of $p_{\mathrm{vs}, i}$ have been found, the values of vapour density at the surface of the film $\rho_{\mathrm{vs}, i}$ can be estimated from the ideal gas law as:

$$
\rho_{\mathrm{vs}, i}=\frac{p_{\mathrm{vs}, i} M_{i}}{R_{\mathrm{u}} T_{\mathrm{s}}},
$$


where $R_{\mathrm{u}}$ is the universal gas constant, $T_{\mathrm{s}}$ is the film surface temperature.

Formula (36) allows us to find $\epsilon_{i}$ using the definition of this parameter (see Expression (13)).

Assuming that the convection mass transfer coefficient, inferred from Equation (7), is the same for all species and the contribution of fuel vapour in the ambient gas can be ignored, the evaporation flux of species from the film surface can be estimated as (cf. Formula (8)):

$$
\dot{m}_{\mathrm{f} i}=h_{\mathrm{m}} \rho_{\mathrm{vs}, i} .
$$

Using Expression (37) and taking into account the effect of thermal swelling, the change of film thickness during the time step $\Delta t$ can be estimated as:

$$
\Delta \delta_{0}=-\Delta t \frac{\left|\dot{m}_{\mathrm{f} i}\right|}{\rho\left(\bar{T}_{0}\right)}+\left[\frac{\rho\left(\bar{T}_{0}\right)}{\rho\left(\bar{T}_{1}\right)}-1\right] \delta_{0},
$$

where $\rho\left(\bar{T}_{0}\right)$ is liquid density at the average temperature of the film calculated at the beginning of the time step, $\rho\left(\bar{T}_{1}\right)$ is the same density but calculated at the end of the time step. Note that Expression (38) predicts that the change in film thickness due to swelling is proportional to $\rho\left(\bar{T}_{0}\right) / \rho\left(\bar{T}_{1}\right)$ (cf. the change in droplet radius due to swelling which is proportional to $\left.\left(\rho\left(\bar{T}_{0}\right) / \rho\left(\bar{T}_{1}\right)\right)^{1 / 3}\right)$. The film thickness at the end of the time step is estimated as:

$$
\delta_{1}=\delta_{0}+\Delta \delta_{0} .
$$

All liquid transport and thermodynamic properties were calculated at liquid film average temperatures and compositions. The contribution of vapour to air transport and thermodynamic properties was ignored. These properties were calculated at the reference temperature $\left(T_{\text {ref }}=(2 / 3) T_{s}+(1 / 3) T_{a}\right.$, where $T_{s}$ is the temperature at the surface of the film, $T_{a}$ is the temperature in the ambient gas). Partial pressures of vapour components and specific heats of their evaporation were calculated at film surface temperatures.

\section{Solution algorithm}

These are the main steps of the numerical algorithm:

1. Assume the initial distribution of temperature and mass fractions of species across the liquid film, or use the distributions obtained at the previous time step (in our case all initial distributions are assumed homogeneous).

2. Calculate species partial pressures and molar fractions in the gas phase using Equation (33).

3. Calculate the values of species evaporation rates $\left(\epsilon_{i}\right)$ using Equation (13).

4. Calculate the values of liquid thermal conductivity and other properties for the mixture of species if the liquid is multi-component.

5. Calculate the distribution of temperature inside the film based on Equation (4), using 40 terms in the series. 
6. Calculate the distribution of species inside the film based on Equation (32), using 200 terms in the series.

7. Calculate the change in film thickness using Equation (38); recalculate the film thickness at the end of the time step using Equation (39).

8. In the case of dimensional $x$, recalculate the distributions of temperature and species for the new film thickness (e.g. $T(x)=T\left(x \delta_{1} / \delta_{0}\right)=T(\tilde{x})$, where $\delta_{0,1}$ are film thicknesses at the beginning and the end of the time step, $\tilde{x}$ is the new $x$ used at the second time step). In the case of dimensionless $x / \delta_{0}$, used in our analysis, no recalculation is required.

9. Return to Step 1 and repeat the calculations for the next time step.

\section{Results}

\subsection{Validation of the model}

The model was validated against the experimental data presented in [26] for the evaporation of a film composed of mixtures of isooctane/3-methylpentane (3MP). The results of the comparison between the model predictions and experimental data for three cases, pure isooctane, pure $3 \mathrm{MP}$, and a $50 \% / 50 \%$ mixture of isooctane and 3MP, are shown in Fig. 1. The same input parameters as in [26] $\left(T_{g}=T_{w}=302.25 \mathrm{~K}\right.$, $\left.T_{0}(x)=293.15 \mathrm{~K}, \delta_{0}=602.72 \mu \mathrm{m}\right)$ and $h=14 \mathrm{~W} /\left(\mathrm{m}^{2} \mathrm{~K}\right)$ were used in our calculations. The value of $h$ was used as a fitting parameter and was close to the one used in [2] where it was assumed that $h=10$ $\mathrm{W} /\left(\mathrm{m}^{2} \mathrm{~K}\right)$.

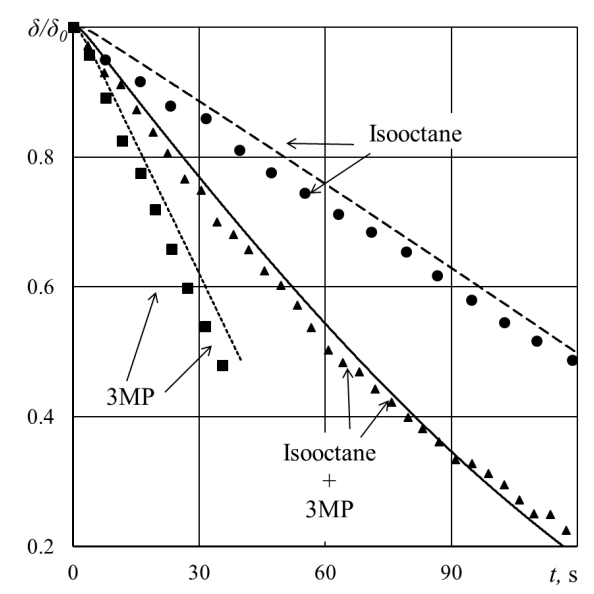

Figure 1: Time evolution of the normalised film thickness. Circles, triangles and squares show the values found in [26]; solid and dashed curves show the corresponding predictions of the model. Three cases were considered: pure isooctane, pure 3MP, and a $50 \% / 50 \%$ mixture of isooctane and 3MP. 
As can be seen from Fig. 1, the results predicted by the new model are reasonably close to the experimental data for all three cases. This gives us confidence to apply the model to the analysis of other films including those observed for conditions relevant to combustion systems.

The results of the comparison between the model predictions and experimental data for time evolution of the average mass fraction of $3 \mathrm{MP}$ in the $50 \% / 50 \%$ mixture of isooctane and $3 \mathrm{MP}$ are shown in Fig. 2. As can be seen from this figure, the agreement between the model predictions and experimental data is good at the initial stage of film heating and evaporation, but at a later stage (after approximately 40 s) the model and experimental data predict different trends. The loss of linearity of the experimental plot shown in Fig. 2 was attributed by the authors of [26] to the violation of Raoult's law (see Equation (33)). Since our model was based on the assumption that this law is valid, it cannot be used for these times. We anticipate that taking into account the contribution of the activity coefficients to the value of the partial pressures of the vapour components would improve the agreement between the predictions of the model and experimental data at these times.

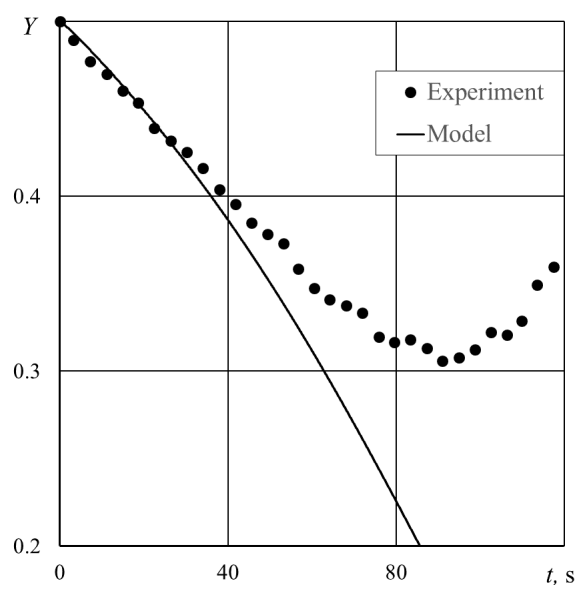

Figure 2: Time evolution of the average mass fraction of 3MP in the case of evaporation of a $3 \mathrm{MP}$ and isooctane mixture film. Solid curve shows the prediction of the model, while dots show experimental data.

In the next section our analysis is focused on the $50 \% / 50 \%$ mixture of heptane $\left(\mathrm{C}_{7} \mathrm{H}_{16}\right)$ and hexadecane $\left(\mathrm{C}_{16} \mathrm{H}_{34}\right)$ which is relevant for automotive applications.

\subsection{Heating and evaporation of a 50\%/50\% heptane and hexadecane film}

The following input parameters, relevant to Diesel injection conditions, were used in our calculations: ambient gas temperature, $T_{\mathrm{g}}=900 \mathrm{~K}$, ambient gas pressure, $p_{\mathrm{g}}=60$ bar, wall temperature, $T_{\mathrm{w}}=500$ 
$\mathrm{K}$, initial film temperature, $T_{0}=363 \mathrm{~K}$, initial film thickness, $\delta_{0}=20 \mu \mathrm{m}$. It was assumed that $h=$ $2000 \mathrm{~W} /\left(\mathrm{m}^{2} \mathrm{~K}\right)$.

The liquid and vapour densities and thermal conductivity were taken from [27]; heat capacity was taken from [25]; saturation vapour pressure was taken from [28, 29]; latent heat of evaporation was taken from [29]; the diffusion coefficient was taken from [27]; liquid diffusivity was taken from [25].

The plot of time evolution of the film thickness is shown in Fig. 3. As can be seen from this figure, initially, the film thickness increases due to thermal swelling and then reduces rather rapidly, which can be attributed to the heating of the surface of the film and evaporation of the most volatile component $\left(\mathrm{C}_{7} \mathrm{H}_{16}\right)$. Then the rate of decrease of this thickness reduces until the evaporation process is completed in just over $25 \mathrm{~ms}$.

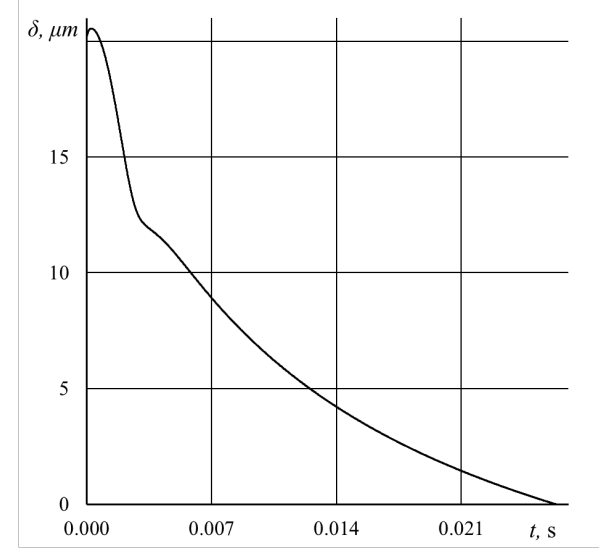

Figure 3: Time evolution of the thickness of the 50\%/50\% heptane and hexadecane film.

The timescale for the completion of the film evaporation shown in Fig. 3 is in agreement with our experimental data presented in [4] for similar operating conditions. Our measurements were performed using high-speed microscopy inside an optical Diesel engine, to observe the liquid films produced at the end of injection around each nozzle orifice. From these observations we estimated that the microscopic liquid films on the tip of the Diesel fuel injector were completely evaporated in approximately $20 \mathrm{~ms}$. Hence the modelled evaporation timescale appears to be consistent with the evaporation of thin fuel films under Diesel engine-like operating conditions.

The plots of time evolution of mass fractions of $\mathrm{C}_{7} \mathrm{H}_{16}$ and $\mathrm{C}_{16} \mathrm{H}_{34}$ are shown in Fig. 4. As can be seen from this figure, $\mathrm{C}_{7} \mathrm{H}_{16}$ totally evaporates in about $4 \mathrm{~ms}$, which is consistent with the results shown in Fig. 3. After that we observe the evaporation of pure $\mathrm{C}_{16} \mathrm{H}_{34}$. 


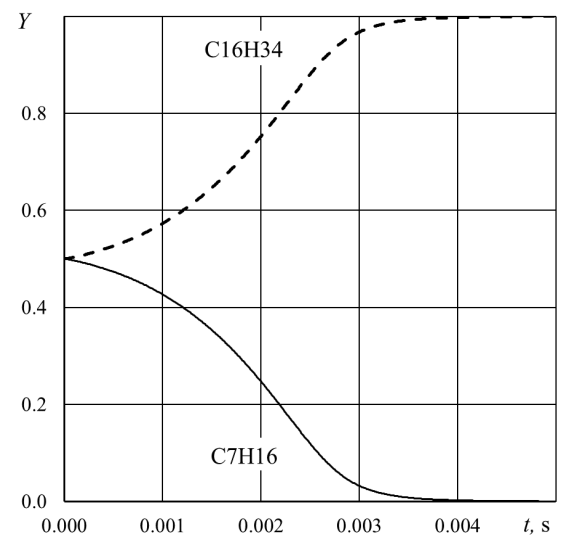

Figure 4: Time evolution of average mass fractions of $\mathrm{C}_{7} \mathrm{H}_{16}$ and $\mathrm{C}_{16} \mathrm{H}_{34}$.

The plots of time evolution of the surface and average temperatures of the film are shown in Fig. 5. As can be seen from this figure, initially both surface and average temperatures rapidly increase with time due to film heating by both hot ambient gas and the hot wall. Once the average temperature of the film exceeds $500 \mathrm{~K}$, the wall turns into a heat sink, and the increase in both surface and average temperatures slows down. Eventually, they reach their maximal values and begin to decrease until both temperatures reach the wall temperature $(500 \mathrm{~K})$ just before the film completely evaporates.

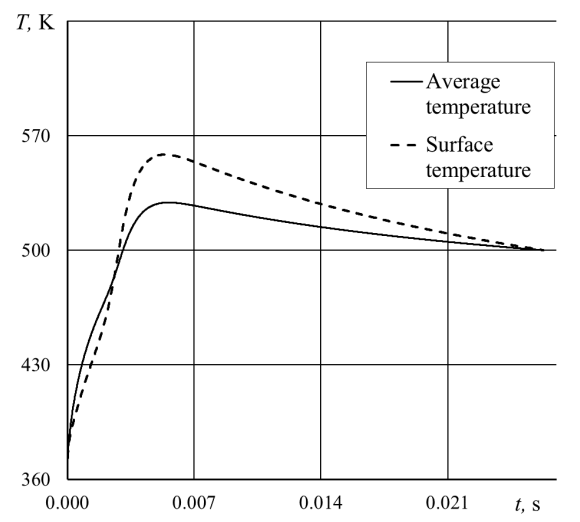

Figure 5: Time evolution of surface and average film temperatures.

\section{Conclusions}

A new model for heating and evaporation of a multi-component one-dimensional thin liquid film is suggested. This model is based on the analytical solutions to the heat transfer and species diffusion equations 
inside the film. For the heat transfer equation, the Dirichlet boundary condition was used at the wall, and the Robin boundary condition was used at the film surface. For the species diffusion equations, the Neumann boundary conditions were used at the wall, and Robin boundary condition, supplemented by Raoult's law, was used at the film surface. The convective heat transfer coefficient is assumed to be constant and the convective mass transfer coefficient is inferred from the Chilton-Colburn analogy. The effect of thermal swelling is taken into account.

The model is validated using previously published experimental data referring to heating and evaporation of a film composed of mixtures of isooctane/3-methylpentane (3MP). It is shown that the predicted time evolutions of thicknesses of pure isooctane, pure 3MP, and a 50\%/50\% isooctane and 3MP films are close to those observed experimentally. The initially observed decrease in the mass fraction of $3 \mathrm{MP}$ in the $50 \% / 50 \%$ mixture of isooctane and 3MP film is shown to be close to the one predicted by the model.

Also the model is applied to the analysis of heating and evaporation of a film composed of $50 \% / 50 \%$ mixture of n-heptane and n-hexadecane at operating conditions relevant to Diesel engines. It is shown that initially the film thickness decreases rather rapidly until all n-heptane has evaporated. Following this, the rate at which the film thickness decreases slows down. The film average and surface temperatures are shown to increase rapidly at the initial stage of film heating and evaporation until these temperatures reach the maximal values. Then both temperatures slowly decrease until they reach the wall temperature just before the film completely evaporates. The predicted evaporation timescale was found to be consistent with our previous measurements of thin fuel films under Diesel engine-like operating conditions.

\section{Acknowledgements}

This work was supported by the UK Engineering and Physical Science Research Council [grants EP/K020528/1 and EP/M002608]. The authors are grateful to Dr Ming Jia (Dalian University of Technology, China) for useful discussion of the results presented in [2].

\section{References}

[1] A. Faghri, Y. Zhang, Transport Phenomena in Multiphase Systems, Elsevier, Amsterdam, 2006.

[2] Y. Zhang, M. Jia, P. Yi, H. Liu, M. Xie, An efficient liquid film vaporization model for multicomponent fuels considering thermal and mass diffusions, Applied Thermal Engineering 112 (2017) 534 - 548. doi:10.1016/j.applthermaleng.2016.10.046.

URL http://www.sciencedirect.com/science/article/pii/S1359431116322736

[3] M. C. Drake, T. D. Fansler, Piston fuel films as a source of smoke and hydrocarbon emissions from a wall-controlled spark-ignited direct-injection engine, in: SAE Technical Report 2003-01-0547.

URL http://papers.sae.org/2003-01-0547 
[4] J. E. Turner, V. Stetsyuk, C. Crua, R. J. Pearson, M. R. Gold, The effect of operating conditions on post-injection fuel discharge in an optical engine, in: ICLASS 13th Triennial International Conference, Tainan, Taiwan, August 23-27, 2015.

[5] H. Sandquist, I. Denbratt, F. Owrang, J. Olsson, Influence of fuel parameters on deposit formation and emissions in a direct injection stratified charge SI engine, in: International Spring Fuels \& Lubricants Meeting, Orlando, Florida, May 7-9, 2001. doi:10.4271/2001-01-2028.

[6] R. E. Olsen, M. C. Ingham, G. M. Parsons, A fuel additive concentrate for removal of injector deposits in light-duty diesels, in: SAE International Fall Fuels \& Lubricants Meeting and Exhibition, Fairborn, OH, October 20-23, 1984. doi:10.4271/841349.

[7] R. Caprotti, W. J. Fowler, G. Lepperhoff, M. Houben, Diesel additive technology effects on injector hole erosion/corrosion, injector fouling and particulate traps, in: SAE International Fuels \& Lubricants Meeting \& Exposition, Philadelphia, Pennsylvania, October 18-21, 1993. doi:10.4271/932739.

[8] G. Lepperhoff, M. Houben, Mechanisms of deposit formation in internal combustion engines and heat exchangers, in: International Congress \& Exposition, Detroit, Michigan, October 17-20, 1993. doi:10.4271/931032.

[9] A. Birgel, N. Ladommatos, P. Aleiferis, S. Zülch, N. Milovanovic, V. Lafon, A. Orlovic, P. Lacey, P. Richards, Deposit formation in the holes of diesel injector nozzles: A critical review, in: Powertrains, Fuels \& Lubricants Meeting, Rosemont, United States, October 6-9, 2008. doi:10.4271/2008-01-2383.

[10] R. Caprotti, A. Breakspear, O. Graupner, T. Klaua, Detergency requirements of future diesel injection systems, in: Powertrain \& Fluid Systems Conference \& Exhibition, San Antonio, TX, October 24-27, 2005. doi:10.4271/2005-01-3901.

[11] B. Argueyrolles, S. Dehoux, P. Gastaldi, L. Grosjean, F. Levy, A. Michel, D. Passerel, Influence of injector nozzle design and cavitation on coking phenomenon, in: International Fuels \& Lubricants Meeting, Kyoto, July 23-27, 2007. doi:10.4271/2007-01-1896.

[12] J. Barker, C. Snape, D. Scurr, Information on the aromatic structure of internal diesel injector deposits from time of flight secondary ion mass spectrometry (ToF-SIMS), in: SAE 2014 World Congress \& Exhibition, Detroit, Michigan, USA, April 8-10, 2014. doi:10.4271/2014-01-1387.

[13] J. Reid, S. Cook, J. Barker, Internal injector deposits from sodium sources, SAE International J. Fuels Lubricants 7 (2) (2014) 436-44. doi:10.4271/2014-01-1388.

[14] P. O'Rourke, A. Amsden, A particle numerical model for wall film dynamics in port-injected engines, SAE Paper 961961 (1996) .

[15] S. Sazhin, Droplets and Sprays, Springer, Heidelberg, 2014.

[16] S. Liye, Z. Weizheng, Z. Tien, Q. Zhaoju, A new approach to transient evaporating film heating modeling based on analytical temperature profiles for internal combustion engines, International Journal of Heat and Mass Transfer 81 (2015) 465 - 469. doi:10.1016/j.ijheatmasstransfer.2014.10.061.

URL http://www.sciencedirect.com/science/article/pii/S0017931014009570

[17] Y. Yan, H. Liu, M. Jia, M. Xie, H. Yin, A one-dimensional unsteady wall film evaporation model, International Journal of Heat and Mass Transfer 88 (2015) 138 - 148. doi:10.1016/j.ijheatmasstransfer.2015.04.082.

URL http://www.sciencedirect.com/science/article/pii/S0017931015004524

[18] D. Stanton, C. Rutland, Multi-dimensional modeling of thin liquid films and spray-wall interactions resulting from impinging sprays, International Journal of Heat and Mass Transfer 41 (1996) 3037-3054.

[19] S. S. Sazhin, P. A. Krutitskii, W. A. Abdelghaffar, E. M. Sazhina, S. V. Mikhalovsky, S. T. Meikle, M. R. Heikal, Transient heating of diesel fuel droplets, International Journal of Heat and Mass Transfer 47 (2004) 3327-3340.

[20] Y. Zhang, M. Jia, H. Liu, M. Xie, Development of an improved liquid film model for spray/wall interaction under enginerelevant conditions, International Journal of Multiphase Flow 79 (2016) 74-87. doi:10.1016/j.ijmultiphaseflow.2015.10.002. URL http://www.sciencedirect.com/science/article/pii/S0301932215002244 
[21] P. T. Tsilingiris, Modeling heat and mass transport phenomena at higher temperatures in solar distillation systems - the Chilton-Colburn analogy, Solar Energy 84 (2010) 308-317.

[22] C. Rakopoulos, G. Mavropoulos, Experimental evaluation of local instantaneous heat transfer characteristics in the combustion chamber of air-cooled direct injection diesel engine, Energy 33 (2008) 1084-1099.

[23] H.-J. Steeman, A. Janssens, M. D. Paepe, On the applicability of the heat and mass transfer analogy in indoor air flows, International Journal of Heat and Mass Transfer 52 (2009) 1431-1442.

[24] A. E. Elwardany, I. G. Gusev, G. Castanet, F. Lemoine, S. S. Sazhin, Mono- and multi-component droplet cooling/heating and evaporation: comparative analysis of numerical models, Atomization and Sprays 21 (11) (2011) 907-931.

[25] S. S. Sazhin, M. A. Qubeissi, R. Nasiri, V. M. Gunko, A. E. Elwardany, F. Lemoine, F. Grisch, M. R. Heikal, A multidimensional quasi-discrete model for the analysis of diesel fuel droplet heating and evaporation, Fuel 129 (2014) 238 - 266. doi:10.1016/j.fuel.2014.03.028.

URL http://www.sciencedirect.com/science/article/pii/S0016236114002695

[26] P. Kelly-Zion, C. Jelf, C. Pursell, S. Oxley, Measuring the changing composition and mass of evaporating fuel films, in: ASME Proceedings ICEF2006, 2006, pp. 77 - 86. doi:10.1115/ICEF2006-1516.

URL http://proceedings.asmedigitalcollection.asme.org/proceeding.aspx?articleID=1596327

[27] M. A. Qubeissi, S. Sazhin, J. Turner, S. Begg, C. Crua, M. Heikal, Modelling of gasoline fuel droplet heating and evaporation, Fuel 159 (2015) 373 - 384. doi:10.1016/j.fuel.2015.06.028.

URL http://www.sciencedirect.com/science/article/pii/S0016236115006080

[28] B. E. Poling, J. M. Prousnitz, J. O'Connell, The Properties of Gases and Liquids, McGraw-Hill, New York, 2001.

[29] C. L. Yaws, Thermophysical Properties of Chemicals and Hydrocarbons, William Andrew Inc., 2008.

[30] I. Gradshtein, I. Ryzhik, Tables of Integrals, Sums, Series and Products, Fizmatgiz (in Russian), Moscow, 1962.

\section{Appendix 1}

The Sturm-Liouville problem for $p<0$

Assuming that $p=-\lambda^{2}<0$ we can write the general solution to Equation (20) as:

$$
v(x)=A \cosh \left(\lambda \frac{x}{\delta_{0}}\right)+B \sinh \left(\lambda \frac{x}{\delta_{0}}\right),
$$

where $A$ and $B$ are arbitrary constants.

The boundary condition at $x=0$ (see (21)) implies that $B=0$. The boundary condition at $x=\delta_{0}$ (see (22)) leads to the following equation:

$$
\frac{A}{\delta_{0}}\left(\lambda \sinh \lambda-\frac{\left|\dot{\delta}_{0}\right| \delta_{0}}{D_{1}} \cosh \lambda\right)=0 .
$$

$A$ in this equation is not equal to zero as we do not consider the trivial solution $v=0$. Hence, Equation (41) can be re-written as:

$$
\operatorname{coth} \lambda=\frac{\lambda D_{1}}{\left|\dot{\delta}_{0}\right| \delta_{0}}
$$


It is easy to show that Equation (42) has two solutions $\pm \lambda_{0}$ for all $\frac{D_{1}}{\left|\dot{\delta}_{0}\right| \delta_{0}}$. These solutions lead to Solutions (40) (eigen functions) which differ only by the sign of $A$. Since the value of the coefficient $A$ is determined by the normalisation condition only (see below), the solution $\lambda=-\lambda_{0}$ can be disregarded. Hence, we can conclude that the solution to Equation (42) gives only one eigenvalue $\lambda=\lambda_{0}>0$ and the corresponding eigen function

$$
v_{0}(x)=\cosh \left(\lambda_{0} \frac{x}{\delta_{0}}\right)
$$

where the normalisation leading to $A=1$ has been chosen.

This result is different from the one described in Appendix H of [15] for spherical droplets.

The direct calculation of the integrals leads to the following expression for the norm of $v_{0}$ :

$$
\left\|v_{0}\right\|^{2}=\int_{0}^{\delta_{d}} v_{0}^{2}(x) \mathrm{d} x=\frac{\delta_{0}}{2}\left[1+\frac{\sinh \left(2 \lambda_{0}\right)}{2 \lambda_{0}}\right] .
$$

The Sturm-Liouville problem for $p>0$

Assuming that $p=\lambda^{2}>0$ we write the general solution to Equation (20) as:

$$
v(x)=A \cos \left(\lambda \frac{x}{\delta_{0}}\right)+B \sin \left(\lambda \frac{x}{\delta_{o}}\right)
$$

where $A$ and $B$ are arbitrary constants.

The boundary condition at $x=0$ (see (21)) implies that $B=0$. The boundary condition at $x=\delta_{0}$ leads to the following equation:

$$
\frac{A}{\delta_{0}}\left(-\lambda \sin \lambda-\frac{\delta_{0}\left|\dot{\delta}_{0}\right|}{D_{\mathrm{l}}} \cos \lambda\right)=0 .
$$

$A$ in this equation is not equal to zero as we do not consider the trivial solution $v=0$. Hence, Equation (46) can be re-written as:

$$
\cot \lambda=-\frac{\lambda D_{1}}{\delta_{0}\left|\dot{\delta}_{0}\right|} .
$$

As in the case $p<0$ we disregard the solutions to this equation corresponding to negative $\lambda$. A countable set of positive solutions to this equation (positive eigenvalues) $\lambda_{n}$ are arranged in ascending order:

$$
0<\lambda_{1}<\lambda_{2}<\ldots<\lambda_{n}
$$

The corresponding eigen functions are presented as:

$$
v_{n}(x)=\cos \left(\lambda_{n} \frac{x}{\delta_{0}}\right)
$$

where the normalisation leading to $A=1$ is chosen as in the case $p<0$.

The direct calculation of the integrals, taking into account Condition (47), leads to the following expression for the norm of $v_{n}$ for $n \geq 1$ :

$$
\left\|v_{n}\right\|^{2}=\int_{0}^{\delta_{0}} v_{n}^{2}(x) \mathrm{d} x=\frac{\delta_{0}}{2}\left[1+\frac{\sin \left(2 \lambda_{n}\right)}{2 \lambda_{n}}\right] .
$$


The orthogonality of functions $v_{n}$ is proven in Appendix 2. We do not provide the proof of completeness of this set of functions. This completeness implicitly follows from the agreement of the results inferred from Expansion (19) with those inferred from direct numerical solution of the species diffusion equation.

Note that following [15] we refer to $\lambda_{n}$, rather than $p$, as eigenvalues of the Sturm-Liouville problem.

\section{Appendix 2}

\section{Proof of Orthogonality of $v_{n}(x)$ for $n \geq 0$}

Let us first consider $n \geq 1$ and calculate the integrals:

$$
I_{n m} \equiv \int_{0}^{\delta_{0}} v_{n}(x) v_{m}(x) \mathrm{d} x=\int_{0}^{\delta_{0}} \cos \left(\lambda_{n} \frac{x}{\delta_{0}}\right) \cos \left(\lambda_{m} \frac{x}{\delta_{0}}\right) \mathrm{d} x=\delta_{0} \int_{0}^{1} \cos \left(\lambda_{n} y\right) \cos \left(\lambda_{m} y\right) \mathrm{d} y .
$$

The integral on the right hand side of this formula is calculated using a simplified version of Formula 2.533(5) of [30] which leads us to:

$$
I_{n m}=\frac{\delta_{0}}{2}\left[\frac{\sin \left(\lambda_{n}+\lambda_{m}\right)}{\lambda_{n}+\lambda_{m}}+\frac{\sin \left(\lambda_{n}-\lambda_{m}\right)}{\lambda_{n}-\lambda_{m}}\right] .
$$

For $\lambda_{n}=\lambda_{m}$, Expression (50) reduces to (49). In the case when $\lambda_{n} \neq \lambda_{m}$ it can be rearranged as:

$$
I_{n m}=\frac{\delta_{0}}{\lambda_{n}^{2}-\lambda_{m}^{2}}\left[\lambda_{n} \sin \lambda_{n} \cos \lambda_{m}-\lambda_{m} \sin \lambda_{m} \cos \lambda_{n}\right] .
$$

Remembering (47), this expression can be further rearranged as:

$$
I_{n m}=\frac{\dot{\delta}_{0} \delta_{0}^{2}}{D_{1}\left(\lambda_{n}^{2}-\lambda_{m}^{2}\right)}\left[\cos \lambda_{n} \cos \lambda_{m}-\cos \lambda_{m} \cos \lambda_{n}\right]=0 .
$$

This completes the proof of orthogonality of $v_{n}(x)$ for $n \geq 1$.

To prove the orthogonality of $v_{0}(x)$ and $v_{n}(x)$ for $n \geq 1$ we calculate the following integral:

$$
I_{0 n}=\int_{0}^{\delta_{0}} \cosh \left(\lambda_{0} \frac{x}{\delta_{0}}\right) \cos \left(\lambda_{n} \frac{x}{\delta_{0}}\right) \mathrm{d} x
$$

where $n \geq 1$.

Using integration by parts twice when calculating the integral on the right hand side of Equation (51) we rearrange this equation as:

$$
I_{0 n}=\frac{\delta_{0}}{\lambda_{n}}\left[\cosh \lambda_{0} \sin \lambda_{n}-\frac{\lambda_{0}}{\lambda_{n}} \sinh \lambda_{0} \cos \lambda_{n}-\frac{\lambda_{0}^{2}}{\lambda_{n} \delta_{0}} I_{0 n}\right],
$$

where $I_{0 n}$ on the right hand side of this equation is the same as in (51).

Equation (52) can be rearranged as:

$$
I_{0 n}=\frac{\frac{\delta_{d}}{\lambda_{n}}\left[\cosh \lambda_{0} \sin \lambda_{n}+\frac{\lambda_{0}}{\lambda_{n}} \sinh \lambda_{0} \cos \lambda_{n}\right]}{1+\left(\frac{\lambda_{0}}{\lambda_{n}}\right)^{2}} .
$$


Remembering Equations (42) and (47), we can see that $I_{0 n}$ defined by Equation (53) is equal to zero. This implies that functions $v_{n}$ are orthogonal for $n \geq 0$ and we can write:

$$
\int_{0}^{\delta_{0}} v_{n}(x) v_{m}(x) \mathrm{d} x=\delta_{n m}\left\|v_{n}\right\|^{2},
$$

where $n \geq 0$ and $m \geq 0,\left\|v_{n}\right\|^{2}$ is defined by Expression (44) when $n=0$ and Expression (49) when $n \geq 1$. 